\title{
Physical activity: the earlier the better. Bones awareness
}

\author{
Iris Iglesia Altaba ${ }^{1,2}$, Pilar Ferrer Santos ${ }^{1}$, María Luisa Miguel-Berges ${ }^{3}$, \\ Paloma Flores Barrantes ${ }^{3}$, Pilar Samper Villagrasa ${ }^{4,5}$ and Gerardo Rodríguez Martínez ${ }^{6,4}$ \\ ${ }^{1}$ Growth, Exercise, Nutrition and Development (GENUD) Research Group, Universidad de Zaragoza Instituto de \\ Investigación Sanitaria Aragón (IIS Aragón), Zaragoza, Spain, \\ ${ }^{2}$ Red de Salud Materno Infantil y del Desarrollo (SAMID), Instituto de Salud Carlos III, Madrid, Spain, \\ ${ }^{3}$ Growth, Exercise, Nutrition and Development (GENUD) Research Group, Universidad de Zaragoza, España 2 \\ Instituto de Investigación Sanitaria Aragón (IIS Aragón), Zaragoza, Spain, \\ ${ }^{4}$ Growth, Exercise, Nutrition and Development (GENUD) Research Group, Universidad de Zaragoza, Zaragoza, \\ Spain, \\ ${ }^{5}$ Department of Pediatrics, Universidad de Zaragoza, Zaragoza, Spain and \\ ${ }^{6}$ Hospital Clínico Universitario Lozano Blesa, Zaragoza, Spain
}

Introduction: There is scarce research on bone health in early stages due to the difficulties in accessing to bone health assessment methods in absence of pathology conditions. Consequently, there is no much information on the determinants of bone health. The aim of this study is to elucidate the association of moderate-vigorous physical activity (MVPA) and bone mineral density of children from Aragon accounting for socioeconomic, role parental modelling, dietary and sedentary behavior effects.

Material \& Methods: The longitudinal cohort 'Growth and Feeding during Lactation and Early Childhood in Children of Aragon (CALINA in Spanish)' included 1,629 born children from Aragon (Spain) in 2009. From those, after 7 years, 339 children (176 boys and 163 girls) were assessed. Bone mineral density (BMD) using Dual X-ray absorciometry (-DXA-), diet quality index (DQI) using a food frequency questionnaire(-FFQ-), sedentary behaviors by questionnaire, and physical activity (using accelerometry-actigraph 3GTX-) were evaluated, as well as if they were or not rapid weight gainers during the first year of life.

Results: From the 339 children, 116 boys $(66.5 \%)$ and 63 girls $(38.7 \%)$ met the current MVPA recommendations of at least? 1 hour/ day. Boys meeting MVPA recommendations did a mean of 84.02 minutes of MVPA per day and had a BMD of $0.60 \mathrm{~g} / \mathrm{cm}^{2}$, while those not meeting the recommendations, did a mean of 43.91 minutes of MVPA and had a BMD of $0.59 \mathrm{~g} / \mathrm{cm}^{2}$. Girls meeting MVPA recommendation did 75.52 minutes and had a BMD of $0.59 \mathrm{~g} / \mathrm{cm}^{2}$, and those not meeting, did $42.81 \mathrm{minutes}$ of MVPA and had a BMD of 0.58. Models, include BMI z-score (age/sex adjusted based in Anthro standards from WHO), weekly sedentary time, DQI, BMI of the mother and whether or not they had been rapid weight gainers from $0-12$ months as confounders and were performed for BMD having MVPA as a predictor. A significant association was found only for boys between MVPA and BMD ( $\beta=0.145$, p-value $=0.02)$ while no association was found for girls $(\beta=0.06$, $\mathrm{p}$-value $=0.40)$.

Discussion: There is still an important physical activity gap between boys and girls. In boys, physical activity is positively associated with BMD, whereas in girls, no significant associations seem to exist. More research is required to elucidated the effects of lifestyle behaviors on bone health in children.

\section{Conflict of Interest}

There is no conflict of interest 